\title{
A stable, high order accurate and efficient hybrid method for flow calculations in complex geometries
}

\author{
Oskar Ålund and J an Nordström
}

\section{Conference article}

Cite this conference article as:

Ålund, O., Nordström, J . A stable, high order accurate and efficient hybrid method for flow calculations in complex geometries, In 2018 AIAA Aerospace Sciences

Meeting, AIAA SciTech Forum, (AIAA 2018-1096), American Institute of Aeronautics and Astronautics; 2018, pp. 1-9. ISBN: 9781624105241,9781510857032

DOI: https:// doi.org/ 10.2514/ 6.2018-1096

Copyright: American Institute of Aeronautics and Astronautics

The self-archived postprint version of this conference article is available at Linköping University Institutional Repository (DiVA):

http:// urn.kb.se/ resolve?urn=urn:nbn:se:liu:diva- 154082 


\title{
A Stable, High Order Accurate and Efficient Hybrid Method for Flow Calculations in Complex Geometries
}

\author{
Oskar Ålund and Jan Nordström \\ Department of Mathematics, Computational Mathematics, Linköping University \\ SE-581 83, Linköping, Sweden
}

\begin{abstract}
The suitability of a discretization method is highly dependent on the shape of the domain. Finite difference schemes are typically efficient, but struggle with complex geometry, while finite element methods are expensive but well suited for complex geometries. In this paper we propose a provably stable hybrid method for a $2 \mathrm{D}$ advection-diffusion problem, using a class of inner product compatible projection operators to couple the non-conforming grids that arise due to varying the discretization method throughout the domain.
\end{abstract}

\section{Introduction}

The hybrid methods envisioned in this paper work by subdividing the spatial domain and applying different methods of discretizations on different subdomains. Earlier investigations of such methods can be found in $17 \sqrt[4]{4}$

It is often too restrictive to require that grid nodes should be collocated at subdomain interfaces. A discretization that uses grids whose nodes are not collocated at the subdomain interfaces is called nonconforming (see for example Figure 1). Recent developments in the study of discretizations on nonconforming grids have made hybrid methods an increasingly popular research area. The groundwork for constructing stable schemes on non-conforming grids was laid in ${ }^{5}$ using so called summation-by-parts preserving interpolation, essentially allowing the scheme to pass information between non-conforming grids in such a way that numerical integral quantities are preserved. This type of preservation is central for stability proofs using numerical differential operators which satisfy the summation-by-parts (SBP) property! A similar but slightly more general technique was proposed in ${ }^{7}$ with the introduction of an intermediary glue grid. The glue grid acts as a form of communication hub for the non-conforming grids. Instead of passing information directly between subdomain grids, solutions on a non-conforming interface are mapped to the glue grid. This is done in an inner product compatible manner, just like in,$\frac{5}{5}$ and carries with it the same benefits in terms of provable stability. The glue grid serves a simplifying purpose both in terms of implementation and scheme design by encapsulating the coupling procedure. This leads to high reusability and greater generality. For example, the code supplied with ${ }^{7}$ was used to construct the projection operators for our proposed scheme with no modification, even though our model problem is different.

In this paper we employ the glue grid technique on non-conforming discretizations of a $2 \mathrm{D}$ advectiondiffusion problem, using SBP operators and simultaneous approximation terms (SATs) ${ }^{6}$

\section{SBP operators and inner product spaces}

Let $\Omega=[-1,1] \times[0,1]$ and consider the subsets $\Omega^{L}=[-1,0] \times[0,1]$ and $\Omega^{R}=[0,1] \times[0,1]$. We discretize the domain $\Omega$ by using equidistant but non-conforming grids on $\Omega^{L}$ and $\Omega^{R}$. More precisely we define the grid points $x_{i}^{L}=-1+i / N_{x}, i=0,1, \ldots, N_{x}$ and $y_{j}^{L}=j / N_{y}, j=0,1, \ldots, N_{y}$ associated to $\Omega^{L}$, and $x_{i}^{R}=i / M_{x}$, $i=0,1, \ldots, M_{x}$ and $y_{j}^{R}=j / M_{y}, j=0,1, \ldots, M_{y}$ associated to $\Omega^{R}$. To the left subdomain $\Omega^{L}$ we associate SBP operators $D_{x}, D_{y}$ in the $x$ - and $y$-directions together with corresponding diagonal norms $P_{x}$ and $P_{y}$. The Kronecker product $\mathbf{P}=P_{x} \otimes P_{y}$ defines an inner product on the space $\mathbb{R}^{\left(N_{x}+1\right)\left(N_{y}+1\right)}$ approximating the standard inner product in $L^{2}\left(\Omega^{L}\right)$. This means that if $u, v \in L^{2}\left(\Omega^{L}\right)$, and $\mathbf{u}, \mathbf{v}$ are vectors formed by 


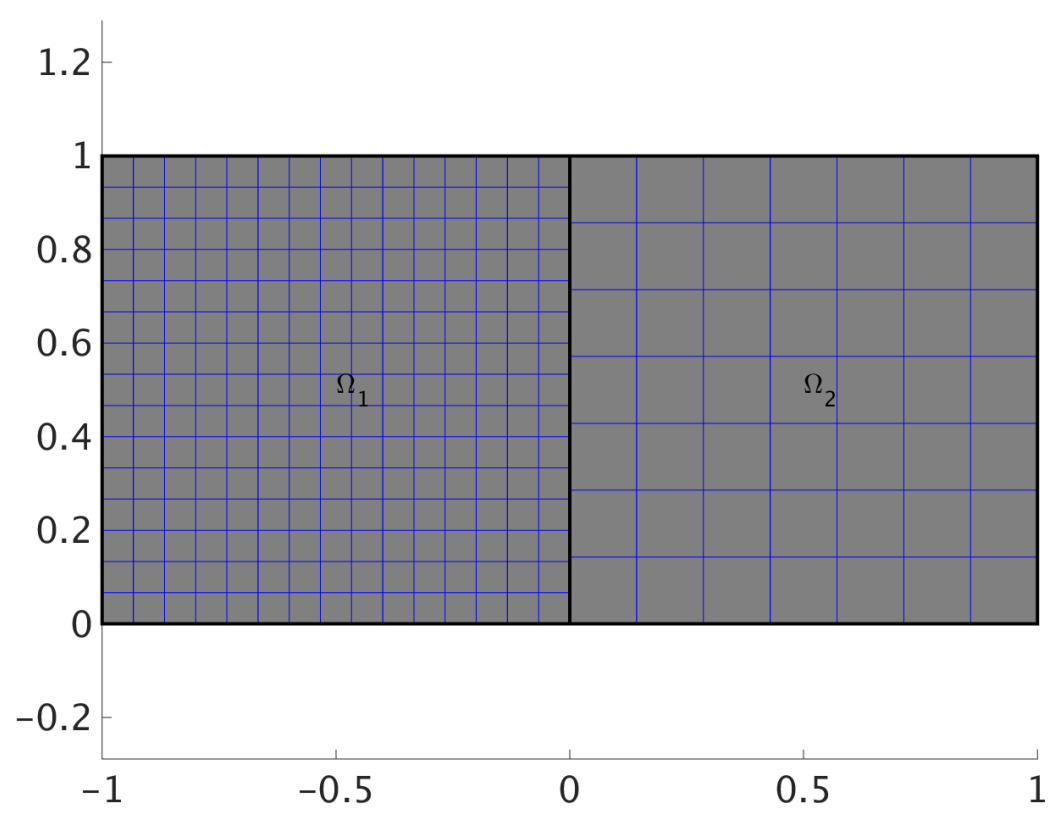

Figure 1. A non-conforming discretization of a rectangular domain $\Omega$.

evaluating $u$ and $v$ at the grid points, then

$$
\langle\mathbf{u}, \mathbf{v}\rangle_{\mathbf{P}}=\mathbf{u}^{\top} \mathbf{P} \mathbf{v} \approx\langle u, v\rangle_{L^{2}}=\iint_{\Omega^{L}} u v d x d y .
$$

Furthermore, 2D SBP operators associated to the inner product $\mathbf{P}$ can be constructed by forming the Kronecker products $D_{x} \otimes I_{y}$ and $I_{x} \otimes D_{y}$, where $I_{x}$ and $I_{y}$ are identity matrices of size $N_{x}$ and $N_{y}$ respectively. The SBP property is inherited in the sense that

$$
\begin{aligned}
& \left\langle\mathbf{u}, \mathbf{D}_{x} \mathbf{v}\right\rangle_{\mathbf{P}}=\langle\mathbf{u}, \mathbf{v}\rangle_{\mathbf{P}_{e}}-\langle\mathbf{u}, \mathbf{v}\rangle_{\mathbf{P}_{w}}-\left\langle\mathbf{D}_{x} \mathbf{u}, \mathbf{v}\right\rangle_{\mathbf{P}} \\
& \left\langle\mathbf{u}, \mathbf{D}_{y} \mathbf{v}\right\rangle_{\mathbf{P}}=\langle\mathbf{u}, \mathbf{v}\rangle_{\mathbf{P}_{n}}-\langle\mathbf{u}, \mathbf{v}\rangle_{\mathbf{P}_{s}}-\left\langle\mathbf{D}_{y} \mathbf{u}, \mathbf{v}\right\rangle_{\mathbf{P}}
\end{aligned}
$$

where $\mathbf{P}_{e}, \mathbf{P}_{w}, \mathbf{P}_{n}, \mathbf{P}_{s}$ are inner products on the boundaries defined as

$$
\mathbf{P}_{e}=E_{N_{x}} \otimes P_{y}, \quad \mathbf{P}_{w}=E_{0} \otimes P_{y}, \quad \mathbf{P}_{n}=P_{x} \otimes E_{N_{y}}, \quad \mathbf{P}_{s}=P_{x} \otimes E_{0} .
$$

Here $E_{i}$ denotes a matrix with a single 1 at position $(i, i)$ and zeros elsewhere.

Similarly, to the right subdomain $\Omega^{R}$ we associate SBP operators $D_{x}, D_{y}$ in the $x$ - and $y$-directions together with corresponding diagonal norms $H_{x}$ and $H_{y}$. Note that while we reuse the notation $D_{x}$ and $D_{y}$ it will be clear from the context which operator is being used. Corresponding 2D SBP operators $\mathbf{D}_{x}, \mathbf{D}_{y}$, and inner products $\mathbf{H}, \mathbf{H}_{e}, \mathbf{H}_{w}, \mathbf{H}_{s}, \mathbf{H}_{n}$ are defined analogously.

Finally we define the discrete gradient and Laplacian,

$$
\nabla \mathbf{u}=\left(\mathbf{D}_{x} \mathbf{u}, \mathbf{D}_{y} \mathbf{u}\right), \quad \Delta \mathbf{u}=\mathbf{D}_{x}^{2} \mathbf{u}+\mathbf{D}_{y}^{2} \mathbf{u} .
$$

\section{Projection operators}

Let $\mathcal{I}=\Omega^{L} \cap \Omega^{R}$ be the interface at which the left and right subdomains meet (see Figure 1). Consider the combined interface grid, called the glue grid ${ }^{7}$ formed by ordering the union $\mathcal{I}_{h}=\left\{y_{j}^{L}\right\} \cup\left\{y_{j}^{R}\right\}$ of the left and right interface grid points. Let $\mathcal{G}_{h} \subset L^{2}(\mathcal{I})$ be the space of piecewise polynomials of order $q_{i}-1$ on the 
subintervals defined by the grid points in $\mathcal{I}_{h}$, where $q_{i}$ is the interior order of the SBP operators. We denote by $\|\cdot\|$ and $\langle\cdot, \cdot\rangle$ the standard norm and inner product in $L^{2}(\mathcal{I})$, i.e. for $u, v \in L^{2}(\mathcal{I})$,

$$
\langle u, v\rangle:=\int_{\mathcal{I}} u v d y \quad \text { and } \quad\|u\|:=\sqrt{\int_{\mathcal{I}} u^{2} d y} .
$$

Given a basis $\left\{\psi_{i}\right\}_{i=1}^{K}$ in $\mathcal{G}_{h}$ (such a basis can be constructed for example by shifting Legendre polynomials as shown in $\left.{ }^{7}\right)$, the matrix $\mathbf{M}=\left(\int_{\mathcal{I}} \psi_{i} \psi_{j} d y\right)_{i j}$ defines an inner product on $\mathbb{R}^{K}$. If $\mathbf{u}=\left(\bar{u}_{1}, \bar{u}_{2}, \ldots, \bar{u}_{K}\right)$ and $\overline{\mathbf{v}}=\left(\bar{v}_{1}, \bar{v}_{2}, \ldots, \bar{v}_{K}\right)$ are vectors of weights corresponding to some functions $\bar{u}=\sum \bar{u}_{i} \psi_{i}$ and $\bar{v}=\sum \bar{v}_{i} \psi_{i}$ in $\mathcal{G}_{h}$, then $\langle\overline{\mathbf{u}}, \overline{\mathbf{v}}\rangle_{\mathbf{M}}=\langle\bar{u}, \bar{v}\rangle$. Hence we will from now on think of $\mathcal{G}_{h}$ as the space $\mathbb{R}^{K}$ equipped with the inner product $\mathbf{M}$, and elements of $\mathcal{G}_{h}$ will always be denoted in boldface and with an overbar.

Let $\mathbf{P}_{\mathcal{I}}=\mathbf{P}_{e}$ and $\mathbf{H}_{\mathcal{I}}=\mathbf{H}_{w}$ be the inner products on the interface from the left and right grid respectively. The projection operators ${ }^{7}$

$$
\begin{array}{ll}
\mathbf{F}_{12 \mathrm{~g}}: \mathbb{R}^{\left(N_{x}+1\right)\left(N_{y}+1\right)} \rightarrow \mathcal{G}_{h} & \mathbf{F}_{\mathrm{g} 21}: \mathcal{G}_{h} \rightarrow \mathbb{R}^{\left(N_{x}+1\right)\left(N_{y}+1\right)} \\
\mathbf{F}_{\mathrm{r} 2 \mathrm{~g}}: \mathbb{R}^{\left(M_{x}+1\right)\left(M_{y}+1\right)} \rightarrow \mathcal{G}_{h} & \mathbf{F}_{\mathrm{g} 2 \mathrm{r}}: \mathcal{G}_{h} \rightarrow \mathbb{R}^{\left(M_{x}+1\right)\left(M_{y}+1\right)}
\end{array}
$$

are such that if $\mathbf{u} \in \mathbb{R}^{\left(N_{x}+1\right)\left(N_{y}+1\right)}, \mathbf{v} \in \mathbb{R}^{\left(M_{x}+1\right)\left(M_{y}+1\right)}$, and $\overline{\mathbf{u}}, \overline{\mathbf{v}} \in \mathcal{G}_{h}$, then

$$
\begin{aligned}
\left\langle\mathbf{u}, \mathbf{F}_{\mathrm{g} 21} \overline{\mathbf{v}}\right\rangle_{\mathbf{P}_{\mathcal{I}}} & =\left\langle\mathbf{F}_{12 \mathrm{~g}} \mathbf{u}, \overline{\mathbf{v}}\right\rangle_{\mathbf{M}} \\
\left\langle\mathbf{v}, \mathbf{F}_{\mathrm{g} 2 \mathrm{r}} \overline{\mathbf{u}}\right\rangle_{\mathbf{H}_{\mathcal{I}}} & =\left\langle\mathbf{F}_{\mathrm{r} 2 \mathrm{~g}} \mathbf{v}, \overline{\mathbf{u}}\right\rangle_{\mathbf{M}} .
\end{aligned}
$$

The properties (3) and (4) are known as $\mathbf{P}_{e^{-}}$and $\mathbf{H}_{w}$-compatibility and allow us to transfer grid representations of functions to piecewise polynomial representations in a pseudo inner product preserving manner. This is crucial for interface penalty formulations and stability analyses on nonconforming grids as it will allow us to penalize differences in the piecewise polynomial representations of the solutions, and to a large extent rely on stability results from the conforming case.

The identification of $\mathcal{G}_{h}$ and $\mathbb{R}^{K}$ is important for the construction of the projection operators since the properties above can then be formulated as the matrix equalities $\mathbf{P}_{e} \mathbf{F}_{\mathrm{g} 21}=\mathbf{F}_{12 \mathrm{~g}}^{\top} \mathbf{M}$ and $\mathbf{H}_{w} \mathbf{F}_{\mathrm{g} 2 \mathrm{r}}=\mathbf{F}_{\mathrm{r} 2 \mathrm{~g}}^{\top} \mathbf{M}$ (operators satisfying this kind of equality, initially in ${ }^{5}$ under the term $S B P$ preserving, is vital even without the concept of a glue grid). These equations merely ensure inner product compatibility however, and have an infinite number of solutions. To ensure that the operators can be used to formulate consistent schemes, accuracy constraints are applied. For example, the operators $\mathbf{F}_{\text {g2l }}$ and $\mathbf{F}_{\text {g2r }}$ must map polynomials of order up to $q_{i}-1$ to their exact point values in the interior of the left and right interface grids. All the accuracy constraints, and the construction of the operators are given in $]^{[7}$

\section{Advection-Diffusion}

Let $\mathbf{a}=(a, b) \in \mathbb{R}^{2}, T>0$ and consider the following coupled advection-diffusion problem:

$$
\begin{aligned}
& u_{t}+\mathbf{a} \cdot \nabla u=\epsilon \Delta u, \text { for }(x, y) \in \Omega^{L}, 0<t<T, \\
&\left.u\right|_{t=0}=f, \text { in } \Omega^{L}, \\
&-\mathbf{a} \cdot \mathbf{n} u+\epsilon \nabla u \cdot \mathbf{n}=g, \text { on } \partial \Omega_{\text {in }}, \\
& \epsilon \nabla u \cdot \mathbf{n}=h, \text { on } \partial \Omega_{\text {out }}, \\
& v_{t}+\mathbf{a} \cdot \nabla v=\epsilon \Delta v, \text { for }(x, y) \in \Omega^{R}, 0<t<T, \\
&\left.v\right|_{t=0}=f, \text { in } \Omega^{R}, \\
&-\mathbf{a} \cdot \mathbf{n} v+\epsilon \nabla v \cdot \mathbf{n}=g, \text { on } \partial \Omega_{\text {in }}, \\
& \epsilon \nabla v \cdot \mathbf{n}=h, \text { on } \partial \Omega_{\text {out }}, \\
& u=v, \text { on } \mathcal{I}, \\
& \frac{\partial u}{\partial \mathbf{n}}=\frac{\partial v}{\partial \mathbf{n}}, \text { on } \mathcal{I}, \\
& 3 \text { of } 8
\end{aligned}
$$


where $\mathbf{n}$ is the outward pointing unit normal, $\partial \Omega_{\text {in }}=\partial \Omega \cap\{\mathbf{a} \cdot \mathbf{n}<0\}$, and $\partial \Omega_{\text {out }}=\partial \Omega \cap\{\mathbf{a} \cdot \mathbf{n} \geq 0\}$.

The problem (5) is discretized using SBP operators on the nonconforming grids. To impose the interface conditions we use Simultaneous Approximation Terms ${ }^{6}$ (SATs) together with the projection operators. For brevity we assume that the initial and external boundary conditions are implemented in a stable manner and in the following energy analysis we omit terms pertaining to the initial state and external boundaries.

Borrowing notation from, 7 the semi-discrete scheme is formulated as

$$
\begin{aligned}
\mathbf{u}_{t}+\mathbf{a} \cdot \nabla \mathbf{u}-\epsilon \Delta \mathbf{u}= & \sigma_{L}^{I} \mathbf{P}^{-1} \mathbf{P}_{e} \mathbf{F}_{\mathrm{g} 21}(\overline{\mathbf{u}}-\overline{\mathbf{v}}) \\
& +\sigma_{L}^{V} \epsilon \mathbf{P}^{-1} \mathbf{P}_{e} \mathbf{F}_{\mathrm{g} 21}\left(\overline{\mathbf{D}_{x} \mathbf{u}}-\overline{\mathbf{D}_{x} \mathbf{v}}\right) \\
& +c_{L}^{I} \mathbf{P}^{-1} \mathbf{P}_{e}\left(\mathbf{F}_{\mathrm{g} 21} \overline{\mathbf{u}}-\mathbf{u}\right) \\
& +c_{L}^{V} \mathbf{P}^{-1} \mathbf{P}_{e}\left(\mathbf{F}_{\mathrm{g} 21} \overline{\mathbf{D}_{x} \mathbf{u}}-\mathbf{D}_{x} \mathbf{u}\right) \\
\mathbf{v}_{t}+\mathbf{a} \cdot \nabla \mathbf{v}-\epsilon \Delta \mathbf{v}= & \sigma_{R}^{I} \mathbf{H}^{-1} \mathbf{H}_{w} \mathbf{F}_{\mathrm{g} 2 \mathrm{r}}(\overline{\mathbf{v}}-\overline{\mathbf{u}}) \\
+ & \sigma_{R}^{V} \epsilon \mathbf{H}^{-1} \mathbf{H}_{w} \mathbf{F}_{\mathrm{g} 2 \mathrm{r}}\left(\overline{\mathbf{D}_{x} \mathbf{v}}-\overline{\mathbf{D}_{x} \mathbf{u}}\right) \\
& +c_{R}^{I} \mathbf{H}^{-1} \mathbf{H}_{w}\left(\mathbf{F}_{\mathrm{g} 2 \mathrm{r}} \overline{\mathbf{v}}-\mathbf{v}\right) \\
& +c_{R}^{V} \mathbf{H}^{-1} \mathbf{H}_{w}\left(\mathbf{F}_{\mathrm{g} 2 \mathrm{r}} \overline{\mathbf{D}_{x} \mathbf{v}}-\mathbf{D}_{x} \mathbf{v}\right),
\end{aligned}
$$

where overbars indicate that a projection operator has been applied and transferred the grid function to its piecewise polynomial representation (e.g. $\left.\overline{\mathbf{u}}=\mathbf{F}_{12 \mathrm{~g}} \mathbf{u}\right)$. The penalty terms $(6 \mathrm{a}),(6 \mathrm{~b}),(\sqrt{7 \mathrm{a}}),(7 \mathrm{~b})$ are nonconforming 2D generalizations of the penalty terms developed in, $\frac{8}{6}$ wile $(6 \mathrm{c}),(6 \mathrm{~d}),(7 \mathrm{c}),(7 \mathrm{~d})$ are correction terms similar to those used in. ${ }^{7}$ In the stability analysis, the correction penalties will essentially replace interface terms originating from the left-hand side by their corresponding glue grid quantities.

The stability analysis of this scheme can be reduced to the conforming case by using properties (3) and 44. Indeed, by transferring interface terms to the common space $\mathcal{G}_{h}$, the final energy estimate is identical in form to that of the conforming case - the only difference being that all inner products are taken with respect to the piecewise polynomial representations of the solutions.

Proposition 1. There exists a positive number $\xi$ such that the scheme (6)-(7) is stable if

$$
\begin{gathered}
\sigma_{R}^{I}=\sigma_{L}^{I}-a, \quad \sigma_{R}^{V}=\sigma_{L}^{V}+1, \quad \sigma_{L}^{I} \leq \frac{a}{2}-\epsilon \frac{\left(\sigma_{L}^{V}\right)^{2}+\left(\sigma_{R}^{V}\right)^{2}}{4 \xi} \\
c_{L}^{I}=-a / 2, \quad c_{L}^{V}=\epsilon, \quad c_{R}^{I}=a / 2, \quad c_{R}^{V}=-\epsilon .
\end{gathered}
$$

Proof. We multiply equation (6) by $2 \mathbf{u}^{\top} \mathbf{P}$ and equation (7) by $2 \mathbf{v}^{\top} \mathbf{H}$ and list the results line by line. For equation (6), using properties (1), (2), (3), (4), the left-hand side becomes (omitting external boundary terms)

$$
\frac{\partial}{\partial t}\|\mathbf{u}\|_{\mathbf{P}}^{2}+a\|\mathbf{u}\|_{\mathbf{P}_{e}}^{2}-2 \epsilon\left\langle\mathbf{u}, \mathbf{D}_{x} \mathbf{u}\right\rangle_{\mathbf{P}_{e}}+2 \epsilon\left(\left\|\mathbf{D}_{x} \mathbf{u}\right\|_{\mathbf{P}}^{2}+\left\|\mathbf{D}_{y} \mathbf{u}\right\|_{\mathbf{P}}^{2}\right)
$$

The terms $a\|\mathbf{u}\|_{\mathbf{P}_{e}}^{2}$ and $-2 \epsilon\left\langle\mathbf{u}, \mathbf{D}_{x} \mathbf{u}\right\rangle_{\mathbf{P}_{e}}$ must be replaced by their corresponding glue grid quantities. To this end we choose $c_{L}^{I}=-a / 2$ and $c_{L}^{V}=\epsilon$.

Let $\|\cdot\|=\|\cdot\|_{\mathbf{M}}$ and $\langle\cdot, \cdot\rangle=\langle\cdot, \cdot\rangle_{\mathbf{M}}$. The penalties on the right-hand side become

$$
\begin{aligned}
2 \sigma_{L}^{I}\left\langle\mathbf{u}, \mathbf{F}_{\mathrm{g} 21} \overline{\mathbf{u}}\right\rangle_{\mathbf{P}_{e}}-2 \sigma_{L}^{I}\left\langle\mathbf{u}, \mathbf{F}_{\mathrm{g} 21} \overline{\mathbf{v}}\right\rangle_{\mathbf{P}_{e}} & =2 \sigma_{L}^{I}\|\overline{\mathbf{u}}\|^{2}-2 \sigma_{L}^{I}\langle\overline{\mathbf{u}}, \overline{\mathbf{v}}\rangle \\
2 \sigma_{L}^{V} \epsilon\left\langle\mathbf{u}, \mathbf{F}_{\mathrm{g} 21} \overline{\mathbf{D}_{x} \mathbf{u}}\right\rangle_{\mathbf{P}_{e}}-2 \sigma_{L}^{V} \epsilon\left\langle\mathbf{u}, \mathbf{F}_{\mathrm{g} 21} \overline{\mathbf{D}_{x} \mathbf{v}}\right\rangle_{\mathbf{P}_{e}} & =2 \sigma_{L}^{V} \epsilon\left\langle\overline{\mathbf{u}}, \overline{\mathbf{D}_{x} \mathbf{u}}\right\rangle-2 \sigma_{L}^{V} \epsilon\left\langle\overline{\mathbf{u}}, \overline{\mathbf{D}_{x} \mathbf{v}}\right\rangle \\
-a\left\langle\mathbf{u}, \mathbf{F}_{\mathrm{g} 21} \overline{\mathbf{u}}\right\rangle_{\mathbf{P}_{e}}+a\|\mathbf{u}\|_{\mathbf{P}_{e}}^{2} & =-a\|\overline{\mathbf{u}}\|^{2}+a\|\mathbf{u}\|_{\mathbf{P}_{e}}^{2} \\
2 \epsilon\left\langle\mathbf{u}, \mathbf{F}_{\mathrm{g} 21} \overline{\mathbf{D}_{x} \mathbf{u}}\right\rangle_{\mathbf{P}_{e}}-2 \epsilon\left\langle\mathbf{u}, \mathbf{D}_{x} \mathbf{u}\right\rangle_{\mathbf{P}_{e}} & =2 \epsilon\left\langle\overline{\mathbf{u}}, \overline{\mathbf{D}_{x} \mathbf{u}}\right\rangle-2 \epsilon\left\langle\mathbf{u}, \mathbf{D}_{x} \mathbf{u}\right\rangle_{\mathbf{P}_{e}}
\end{aligned}
$$

Here equation (11) and (12) transfers the inner products to $\mathcal{G}_{h}$, and equation (13) and (14) will replace the quantities $a\|\mathbf{u}\|_{\mathbf{P}_{e}}^{2}$ and $2 \epsilon\left\langle\mathbf{u}, \mathbf{D}_{x} \mathbf{u}\right\rangle_{\mathbf{P}_{e}}$ (appearing in the left-hand side expression (10)) with their corresponding quantities in $\mathcal{G}_{h}$. 
Similarly, for equation (7), the left-hand side becomes (omitting external boundary terms)

$$
\frac{\partial}{\partial t}\|\mathbf{v}\|_{\mathbf{H}}^{2}-a\|\mathbf{v}\|_{\mathbf{H}_{w}}^{2}+2 \epsilon\left\langle\mathbf{v}, \mathbf{D}_{x} \mathbf{v}\right\rangle_{\mathbf{H}_{w}}+2 \epsilon\left(\left\|\mathbf{D}_{x} \mathbf{v}\right\|_{\mathbf{H}}^{2}+\left\|\mathbf{D}_{y} \mathbf{v}\right\|_{\mathbf{H}}^{2}\right) .
$$

Again, to replace the terms $-a\|\mathbf{v}\|_{\mathbf{H}_{w}}^{2}$ and $2 \epsilon\left\langle\mathbf{v}, \mathbf{D}_{x} \mathbf{v}\right\rangle_{\mathbf{H}_{w}}$ with their corresponding glue grid quantities, we choose $c_{R}^{I}=a / 2$ and $c_{R}^{V}=-\epsilon$.

The penalties on the right-hand side become

$$
\begin{aligned}
2 \sigma_{R}^{I}\left\langle\mathbf{v}, \mathbf{F}_{\mathrm{g} 2 \mathrm{r}} \overline{\mathbf{v}}\right\rangle_{\mathbf{H}_{w}}-2 \sigma_{R}^{I}\left\langle\mathbf{v}, \mathbf{F}_{\mathrm{g} 2 \mathrm{r}} \overline{\mathbf{u}}\right\rangle_{\mathbf{H}_{w}} & =2 \sigma_{R}^{I}\|\overline{\mathbf{v}}\|^{2}-2 \sigma_{R}^{I}\langle\overline{\mathbf{v}}, \overline{\mathbf{u}}\rangle \\
2 \sigma_{R}^{V} \epsilon\left\langle\mathbf{v}, \mathbf{F}_{\mathrm{g} 2 \mathrm{r}} \overline{\mathbf{D}_{x} \mathbf{v}}\right\rangle_{\mathbf{H}_{w}}-2 \sigma_{R}^{V} \epsilon\left\langle\mathbf{v}, \mathbf{F}_{\mathrm{g} 2 \mathrm{r}} \overline{\mathbf{D}_{x} \mathbf{u}}\right\rangle_{\mathbf{H}_{w}} & =2 \sigma_{R}^{V} \epsilon\left\langle\overline{\mathbf{v}}, \overline{\mathbf{D}_{x} \mathbf{v}}\right\rangle-2 \sigma_{R}^{V} \epsilon\left\langle\overline{\mathbf{v}}, \overline{\mathbf{D}_{x} \mathbf{u}}\right\rangle \\
a\left\langle\mathbf{v}, \mathbf{F}_{\mathrm{g} 2 \mathrm{r}} \overline{\mathbf{v}}\right\rangle_{\mathbf{H}_{w}}-a\|\mathbf{v}\|_{\mathbf{H}_{w}}^{2} & =a\|\overline{\mathbf{v}}\|^{2}-a\|\mathbf{v}\|_{\mathbf{H}_{w}}^{2} \\
-2 \epsilon\left\langle\mathbf{v}, \mathbf{F}_{\mathrm{g}_{21}} \overline{\mathbf{D}_{x} \mathbf{v}}\right\rangle_{\mathbf{H}_{w}}+2 \epsilon\left\langle\mathbf{v}, \mathbf{D}_{x} \mathbf{v}\right\rangle_{\mathbf{H}_{w}} & =-2 \epsilon\left\langle\overline{\mathbf{v}}, \overline{\mathbf{D}_{x} \mathbf{v}}\right\rangle+2 \epsilon\left\langle\mathbf{v}, \mathbf{D}_{x} \mathbf{v}\right\rangle_{\mathbf{H}_{w}}
\end{aligned}
$$

It follows that the rate of change $\frac{\partial}{\partial t}\left(\|\mathbf{u}\|_{\mathbf{P}}^{2}+\|\mathbf{v}\|_{\mathbf{H}}^{2}\right)$ in the energy of the solution satisfies

$$
\begin{aligned}
\frac{\partial}{\partial t}\left(\|\mathbf{u}\|_{\mathbf{P}}^{2}+\|\mathbf{v}\|_{\mathbf{H}}^{2}\right)= & \left(2 \sigma_{L}^{I}-a\right)\|\overline{\mathbf{u}}\|^{2}+\left(2 \sigma_{R}^{I}+a\right)\|\overline{\mathbf{v}}\|^{2} \\
+ & 2 \epsilon\left(\sigma_{L}^{V}+1\right)\left\langle\overline{\mathbf{u}}, \overline{\mathbf{D}_{x} \mathbf{u}}\right\rangle+2 \epsilon\left(\sigma_{R}^{V}-1\right)\left\langle\overline{\mathbf{v}}, \overline{\mathbf{D}_{x} \mathbf{v}}\right\rangle \\
& -2\left(\sigma_{L}^{I}+\sigma_{R}^{I}\right)\langle\overline{\mathbf{u}}, \overline{\mathbf{v}}\rangle-2 \sigma_{L}^{V} \epsilon\left\langle\overline{\mathbf{u}}, \overline{\mathbf{D}_{x} \mathbf{v}}\right\rangle-2 \sigma_{R}^{V} \epsilon\left\langle\overline{\mathbf{v}}, \overline{\mathbf{D}_{x} \mathbf{u}}\right\rangle \\
& -2 \epsilon\left(\left\|\mathbf{D}_{x} \mathbf{u}\right\|_{\mathbf{P}}^{2}+\left\|\mathbf{D}_{y} \mathbf{u}\right\|_{\mathbf{P}}^{2}+\left\|\mathbf{D}_{x} \mathbf{v}\right\|_{\mathbf{H}}^{2}+\left\|\mathbf{D}_{y} \mathbf{v}\right\|_{\mathbf{H}}^{2}\right) .
\end{aligned}
$$

The right-hand side of 15 now consists of glue grid integral quantities, except for the bottom row. However, we can find a bound for the bottom row in terms of glue grid integral quantities. It can be shown that there exists a positive number $\alpha$ depending on the grid resolutions such that $\alpha\left\|\mathbf{D}_{x} \mathbf{u}\right\|_{\mathbf{P}_{e}}^{2} \leq\left\|\mathbf{D}_{x} \mathbf{u}\right\|_{\mathbf{P}}^{2}$ and $\alpha\left\|\mathbf{D}_{x} \mathbf{v}\right\|_{\mathbf{H}_{w}}^{2} \leq\left\|\mathbf{D}_{x} \mathbf{v}\right\|_{\mathbf{H}}^{2}$. Furthermore, using properties (3) and (4), we have

$$
\begin{aligned}
\left\|\overline{\mathbf{D}_{x} \mathbf{u}}\right\|^{2} & =\left\langle\overline{\mathbf{D}_{x} \mathbf{u}}, \overline{\mathbf{D}_{x} \mathbf{u}}\right\rangle \\
& =\left\langle\mathbf{D}_{x} \mathbf{u}, \mathbf{F}_{\mathrm{g} 21} \overline{\mathbf{D}_{x} \mathbf{u}}\right\rangle_{\mathbf{P}_{e}} \\
& =\left\langle\mathbf{D}_{x} \mathbf{u}, \mathbf{F}_{\mathrm{g} 21} \mathbf{F}_{12 \mathrm{~g}} \mathbf{D}_{x} \mathbf{u}\right\rangle_{\mathbf{P}_{e}} \\
& \leq\left\|\mathbf{F}_{\mathrm{g} 21} \mathbf{F}_{12 \mathrm{~g}}\right\|\left\|\mathbf{D}_{x} \mathbf{u}\right\|_{\mathbf{P}_{e}}^{2} .
\end{aligned}
$$

Hence there is a positive number $\xi$ depending on the grid resolutions and the Kozdon-Wilcox operators, such that $\xi\left\|\overline{\mathbf{D}_{x} \mathbf{u}}\right\|^{2} \leq\left\|\mathbf{D}_{x} \mathbf{u}\right\|_{\mathbf{P}}^{2}$ and $\xi\left\|\overline{\mathbf{D}_{x} \mathbf{v}}\right\|^{2} \leq\left\|\mathbf{D}_{x} \mathbf{v}\right\|_{\mathbf{H}}^{2}$. Using these inequalities together with equation (15) gives the estimate

$$
\begin{aligned}
\frac{\partial}{\partial t}\left(\|\mathbf{u}\|_{\mathbf{P}}^{2}+\|\mathbf{v}\|_{\mathbf{H}}^{2}\right) \leq & \left(2 \sigma_{L}^{I}-a\right)\|\overline{\mathbf{u}}\|^{2}+\left(2 \sigma_{R}^{I}+a\right)\|\overline{\mathbf{v}}\|^{2} \\
+ & 2 \epsilon\left(\sigma_{L}^{V}+1\right)\left\langle\overline{\mathbf{u}}, \overline{\mathbf{D}_{x} \mathbf{u}}\right\rangle+2 \epsilon\left(\sigma_{R}^{V}-1\right)\left\langle\overline{\mathbf{v}}, \overline{\mathbf{D}_{x} \mathbf{v}}\right\rangle \\
& -2\left(\sigma_{L}^{I}+\sigma_{R}^{I}\right)\langle\overline{\mathbf{u}}, \overline{\mathbf{v}}\rangle-2 \sigma_{L}^{V} \epsilon\left\langle\overline{\mathbf{u}}, \overline{\mathbf{D}_{x} \mathbf{v}}\right\rangle-2 \sigma_{R}^{V} \epsilon\left\langle\overline{\mathbf{v}}, \overline{\mathbf{D}_{x} \mathbf{u}}\right\rangle \\
& -2 \epsilon \xi\left\|\overline{\mathbf{D}_{x} \mathbf{u}}\right\|^{2}-2 \epsilon \xi\left\|\overline{\mathbf{D}_{x} \mathbf{v}}\right\|^{2} \\
= & \mathbf{w}^{\top} B \otimes \mathbf{M w},
\end{aligned}
$$

where

$$
\mathbf{w}^{\top}=\left(\begin{array}{llll}
\overline{\mathbf{u}}^{\top} & \overline{\mathbf{v}}^{\top} & \overline{\mathbf{D}_{x} \mathbf{u}^{\top}} & \overline{\mathbf{D}_{x} \mathbf{v}}
\end{array}\right),
$$

and

$$
B=\left[\begin{array}{cccc}
\left(-a+2 \sigma_{L}^{I}\right) & -\left(\sigma_{L}^{I}+\sigma_{R}^{I}\right) & \epsilon\left(\sigma_{L}^{V}+1\right) & -\sigma_{L}^{V} \epsilon \\
-\left(\sigma_{L}^{I}+\sigma_{R}^{I}\right) & 2 \sigma_{R}^{I}+a & -\sigma_{R}^{V} \epsilon & \epsilon\left(\sigma_{R}^{V}-1\right) \\
\epsilon\left(\sigma_{L}^{V}+1\right) & -\sigma_{R}^{V} \epsilon & -2 \epsilon \xi & 0 \\
-\sigma_{L}^{V} \epsilon & \epsilon\left(\sigma_{R}^{V}-1\right) & 0 & -2 \epsilon \xi
\end{array}\right]
$$

The matrix $B$ is shown in $\frac{8}{8}$ to be negative semi-definite if $(8)$ holds. 


\section{Initial numerical results}

We test our scheme with the parameters $a=1, b=0.5, \epsilon=0.01$, by pulling data from the manufactured solution $\cos (x+y+t)$. The left and right subdomains are discretized into non-conforming grids like the one in Figure 1. Solutions and $L^{2}$-errors in space are computed at time $T=1$. The first discretization uses a $16 \times 16$ grid in the left subdomain and an $8 \times 8$-grid in the right subdomain. Thereafter the subdomain resolutions are increased and a convergence rate is computed as $\left(\log \left(e_{\text {coarse }}\right)-\log \left(e_{\text {fine }}\right)\right) /\left(\log \left(h_{\text {coarse }}\right)-\log \left(h_{\text {fine }}\right)\right)$. Here $e_{\text {coarse }}=\sqrt{\left\|\mathbf{u}-\mathbf{u}_{\text {exact }}\right\|_{\mathbf{P}}^{2}+\left\|\mathbf{v}-\mathbf{v}_{\text {exact }}\right\|_{\mathbf{H}}^{2}}(\mathbf{u}$ and $\mathbf{v}$ are the approximate solutions on the left and right subdomains, and $\mathbf{u}_{\text {exact }}$ and $\mathbf{v}_{\text {exact }}$ are the exact solution evaluated at the grid nodes) is the error on the coarser discretization, while $h_{\text {coarse }}$ is the grid spacing. $e_{\text {fine }}$ and $h_{\text {fine }}$ is the error and grid spacing on the finer discretization, defined analogously. Note that the spacings $h_{\text {coarse }}$ and $h_{\text {fine }}$ can be taken on either the left or the right subdomain (as long as we are consistent), since the ratio between them is the same in either case. The results for various order SBP operators are listed in the Table 1.

Table 1. $L^{2}$ errors at time $T=1$ and convergence rates for different SBP operators. The format $\operatorname{SBP}(2 p, p)$ means that the operator is of order $2 p$ for interior nodes and $p$ for boundary nodes. The res column displays the resolution in the format $N / M$, meaning the left subdomain uses an $N \times N$-grid and the right subdomain uses an $M \times M$-grid.

\begin{tabular}{|r|l|l|}
\hline \multicolumn{3}{|c}{$\operatorname{SBP}(2,1)$} \\
\hline res & error & rate \\
\hline $16 / 8$ & $6.72 \mathrm{e}-03$ & \\
$32 / 16$ & $1.43 \mathrm{e}-03$ & 2.13 \\
$64 / 32$ & $3.30 \mathrm{e}-04$ & 2.07 \\
$128 / 64$ & $8.13 \mathrm{e}-05$ & 2.00 \\
\hline
\end{tabular}

\begin{tabular}{|r|c|c|}
\hline \multicolumn{3}{|c}{$\operatorname{SBP}(4,2)$} \\
\hline res & error & rate \\
\hline $16 / 8$ & $4.47 \mathrm{e}-03$ & \\
$32 / 16$ & $4.69 \mathrm{e}-04$ & 3.10 \\
$64 / 32$ & $5.31 \mathrm{e}-05$ & 3.07 \\
$128 / 64$ & $6.17 \mathrm{e}-06$ & 3.07 \\
\hline
\end{tabular}

\begin{tabular}{|r|c|c|}
\multicolumn{3}{|c}{$\operatorname{SBP}(6,3)$} \\
\hline res & error & rate \\
\hline $32 / 16$ & $2.96 \mathrm{e}-05$ & \\
$64 / 32$ & $1.43 \mathrm{e}-06$ & 4.27 \\
$96 / 48$ & $2.61 \mathrm{e}-07$ & 4.14 \\
$128 / 64$ & $7.92 \mathrm{e}-08$ & 4.11 \\
\hline
\end{tabular}

\begin{tabular}{|r|c|c|}
\multicolumn{3}{|c}{$\operatorname{SBP}(8,4)$} \\
\hline res & error & rate \\
\hline $32 / 16$ & $5.53 \mathrm{e}-02$ & \\
$64 / 32$ & $1.55 \mathrm{e}-07$ & 18 \\
$96 / 48$ & $1.82 \mathrm{e}-08$ & 5.21 \\
$128 / 64$ & $3.99 \mathrm{e}-09$ & 5.22 \\
\hline
\end{tabular}

\section{Curvilinear grids}

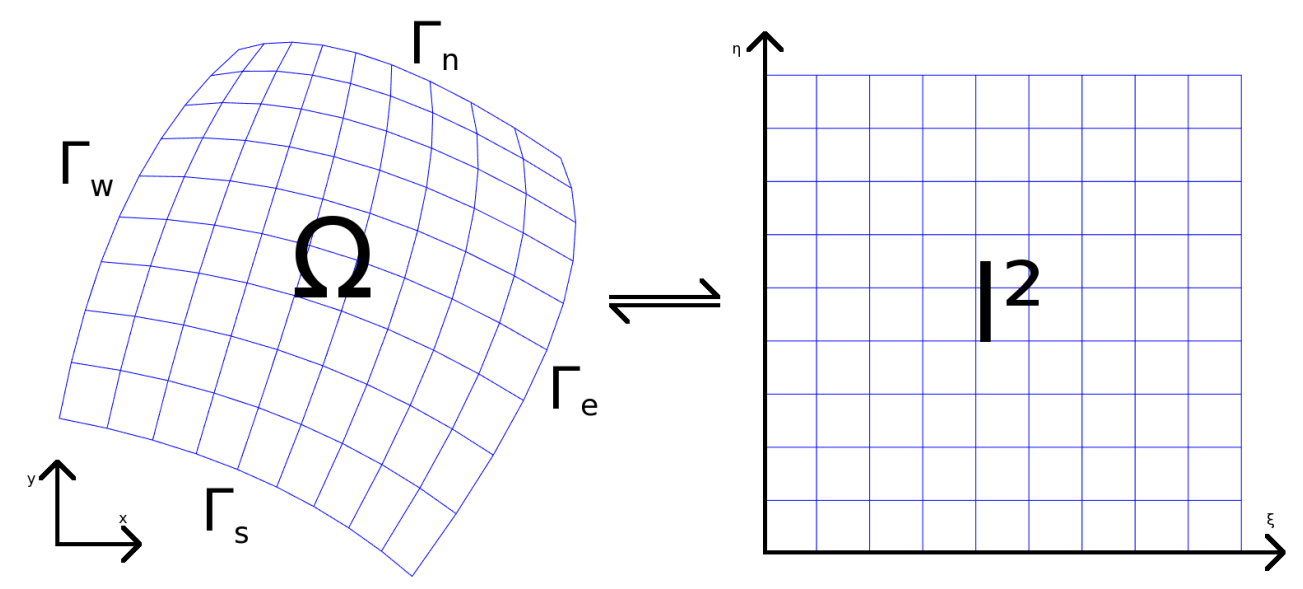

Figure 2. A smooth transformation from curvilinear to square grid.

To be able to handle non-rectangular domains $\Omega$ it is convenient to define SBP operators acting on 
curvilinear grids. This way it is possible to formulate discrete schemes directly on curved domains with the same simplicity as in the rectangular case. That is, the common procedure of transforming curved domains to rectangular domains is an effort that need only be considered once in order to construct appropriate SBP operators. Once these operators have been constructed, there is no need for coordinate transforms in the involved equations, and the stability analysis will perfectly mimic the continuous energy method in the untransformed space.

Consider the curved domain $\Omega$ along with its curvilinear grid $\Omega_{h}$ shown in Figure 2 and let $\Gamma=\partial \Omega$. The construction of curvilinear SBP operators is essentially done by combining standard SBP operators with the coordinate transform Jacobian. This results in a quadrature matrix $\mathbf{P}_{\Omega}$, a boundary quadrature matrix $\mathbf{P}_{\Gamma}$, and numerical partial differential operators $\mathbf{D}_{x}$ and $\mathbf{D}_{y}$ which satisfy a numerical analogue of integration by parts. In a future paper we will develop difference operators that satisfy

$$
\begin{aligned}
\left\langle\mathbf{u}, \mathbf{D}_{x} \mathbf{v}\right\rangle_{\mathbf{P}_{\Omega}} & =\left\langle\mathbf{u}, \mathbf{N}_{x} \mathbf{v}\right\rangle_{\mathbf{P}_{\Gamma}}-\left\langle\mathbf{D}_{x} \mathbf{u}, \mathbf{v}\right\rangle_{\mathbf{P}_{\Omega}} \\
\left\langle\mathbf{u}, \mathbf{D}_{y} \mathbf{v}\right\rangle_{\mathbf{P}_{\Omega}} & =\left\langle\mathbf{u}, \mathbf{N}_{y} \mathbf{v}\right\rangle_{\mathbf{P}_{\Gamma}}-\left\langle\mathbf{D}_{y} \mathbf{u}, \mathbf{v}\right\rangle_{\mathbf{P}_{\Omega}}
\end{aligned}
$$

Here $\mathbf{N}_{x}$ and $\mathbf{N}_{y}$ are outward pointing boundary normals. The relations (17) and (18) mimic the familiar, continuous integration by parts formulas

$$
\left\langle u, v_{x}\right\rangle=\left\langle u, n_{x} v\right\rangle_{L^{2}(\Gamma)}-\left\langle u_{x}, v\right\rangle_{L^{2}(\Omega)} \text { and }\left\langle u, v_{y}\right\rangle=\left\langle u, n_{y} v\right\rangle_{L^{2}(\Gamma)}-\left\langle u_{y}, v\right\rangle_{L^{2}(\Omega)} .
$$

Using these operators, together with curvilinear versions of the projection operators discussed above, it is straightforward to formulate stable schemes also on curved non-conforming grids.

Consider problem (5) posed on the domain in Figure 3. Our discretization has essentially the same form as in the rectangular case, except with a matrix penalty coefficient in the inviscid coupling due to the varying interface normals.

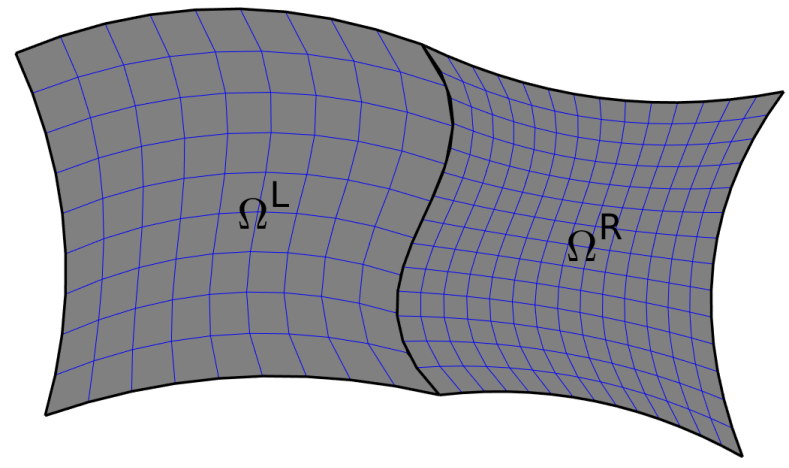

Figure 3. Two curvilinear, non-conforming grids.

Let $\frac{\partial \mathbf{u}}{\partial \mathbf{n}}=\mathbf{N}_{x} \mathbf{D}_{x} \mathbf{u}+\mathbf{N}_{y} \mathbf{D}_{y} \mathbf{u}, \frac{\partial \mathbf{v}}{\partial \mathbf{n}}=\mathbf{N}_{x} \mathbf{D}_{x} \mathbf{v}+\mathbf{N}_{y} \mathbf{D}_{y} \mathbf{v}$. Our scheme is then (omitting boundary penalties)

$$
\begin{aligned}
\mathbf{u}_{t}+\mathbf{a} \cdot \nabla \mathbf{u}-\epsilon \Delta \mathbf{u}= & \mathbf{P}^{-1} \mathbf{P}_{\mathcal{I}} \mathbf{F}_{\mathrm{g} 21} \boldsymbol{\Sigma}_{L}^{I}(\overline{\mathbf{u}}-\overline{\mathbf{v}}) \\
& +\sigma_{L}^{V} \epsilon \mathbf{P}^{-1} \mathbf{P}_{\mathcal{I}} \mathbf{F}_{\mathrm{g} 21}\left(\overline{\frac{\partial \mathbf{u}}{\partial \mathbf{n}}}-\overline{\frac{\partial \mathbf{v}}{\partial \mathbf{n}}}\right) \\
& +c_{L}^{I} \mathbf{P}^{-1} \mathbf{P}_{\mathcal{I}}\left(\mathbf{F}_{\mathrm{g} 21} \overline{(\mathbf{a} \cdot \mathbf{N})} \overline{\mathbf{u}}-(\mathbf{a} \cdot \mathbf{N}) \mathbf{u}\right) \\
& +c_{L}^{V} \mathbf{P}^{-1} \mathbf{P}_{\mathcal{I}}\left(\mathbf{F}_{\mathrm{g} 21} \frac{\overline{\partial \mathbf{u}}}{\partial \mathbf{n}}-\frac{\partial \mathbf{u}}{\partial \mathbf{n}}\right)
\end{aligned}
$$




$$
\begin{aligned}
\mathbf{v}_{t}+\mathbf{a} \cdot \nabla \mathbf{v}-\epsilon \Delta \mathbf{v}= & \mathbf{H}^{-1} \mathbf{H}_{\mathcal{I}} \mathbf{F}_{\mathrm{g} 2 \mathrm{r}} \boldsymbol{\Sigma}_{R}^{I}(\overline{\mathbf{v}}-\overline{\mathbf{u}}) \\
& +\sigma_{R}^{V} \epsilon \mathbf{H}^{-1} \mathbf{H}_{\mathcal{I}} \mathbf{F}_{\mathrm{g} 2 \mathrm{r}}\left(\overline{\frac{\partial \mathbf{v}}{\partial \mathbf{n}}}-\frac{\overline{\partial \mathbf{u}}}{\partial \mathbf{n}}\right) \\
& +c_{R}^{I} \mathbf{H}^{-1} \mathbf{H}_{\mathcal{I}}\left(\mathbf{F}_{\mathrm{g} 2 \mathrm{r}} \overline{(\mathbf{a} \cdot \mathbf{N})} \overline{\mathbf{v}}-(\mathbf{a} \cdot \mathbf{N}) \mathbf{v}\right) \\
& +c_{R}^{V} \mathbf{H}^{-1} \mathbf{H}_{\mathcal{I}}\left(\mathbf{F}_{\mathrm{g} 2 \mathrm{r}} \frac{\overline{\partial \mathbf{v}}}{\partial \mathbf{n}}-\frac{\partial \mathbf{v}}{\partial \mathbf{n}}\right)
\end{aligned}
$$

Here $\overline{\mathbf{a} \cdot \mathbf{N}}=\mathbf{F}_{12 \mathrm{~g}}(\mathbf{a} \cdot \mathbf{N}) \mathbf{F}_{\mathrm{g} 2 \mathrm{l}}$ is the normal velocity transferred to the glue grid.

The full details of the curvilinear case will be supplied in a future paper.

\section{References}

${ }^{1}$ Nordström, J. and Gong, J., "A stable hybrid method for hyperbolic problems," Journal of Computational Physics, Vol. 212, No. 2, 2006, pp. 436-453.

${ }^{2}$ Gong, J. and Nordström, J., "A stable and efficient hybrid scheme for viscous problems in complex geometries," Journal of Computational Physics, Vol. 226, No. 2, 2007, pp. 1291-1309.

${ }^{3}$ Nordström, J. e. a., "A hybrid method for unsteady inviscid fluid flow," Computers $\&$ Fluids, Vol. 38, No. 4, 2009, pp. $875-882$.

${ }^{4} \mathrm{O}$ 'Reilly, O. e. a., "Simulation of earthquake rupture dynamics in complex geometries using coupled finite difference and finite volume methods," Communications in Computational Physics, Vol. 17, No. 2, 2015, pp. 337-370.

${ }^{5}$ Mattsson, K. and Carpenter, M. H., "Stable and accurate interpolation operators for high-order multiblock finite difference methods," SIAM Journal on Scientific Computing, Vol. 32, 2010, pp. 2298-2320.

${ }^{6}$ Svärd, M. and Nordström, J., "Review of summation-by-parts schemes for initial-boundary-value problems," Journal of Computational Physics, Vol. 268, 2014, pp. 17-38.

${ }^{7}$ Kozdon, J. E. and Wilcox, L. C., "Stable Coupling of Nonconforming, High-Order Finite Difference Methods," SIAM Journal on Scientific Computing, Vol. 38, 2016, pp. A923-A952.

${ }^{8}$ Carpenter, M. H., Nordström, J., and Gottlieb, D., "A stable and conservative interface treatment of arbitrary spatial accuracy," Journal of Computational Physics, Vol. 148, 1999, pp. 341-365. 